\title{
Partial persistence of exercise-induced myocardial angiogenesis following 4-week detraining in the rat
}

\author{
Marina Marini · Elisabetta Falcieri • Vittoria Margonato · Davide Treré • \\ Rosa Lapalombella $\cdot$ Simona di Tullio $\cdot$ Cosetta Marchionni $\cdot$ Sabrina Burattini $\cdot$ \\ Michele Samaja $\cdot$ Fabio Esposito $\cdot$ Arsenio Veicsteinas
}

Accepted: 15 December 2007 / Published online: 3 January 2008

(C) Springer-Verlag 2007

\begin{abstract}
Enhanced angiogenesis, or capillary growth, has a prominent role among the various beneficial effects of exercise training on the myocardium. The aim of the present study is to assess if training-induced increases in capillarity and vascularization persist after 4 weeks of detraining. Adult male rats were trained to run on a treadmill for 10 weeks at $\sim 60 \% V \mathrm{O}_{2 \max }$, which did not induce cardiac hypertrophy, but increased $(P<0.05)$ the soleus/ body weight ratio, left ventricle capillarity and von Willebrand-positive cell density $(n=6)$. In another group of
\end{abstract}

M. Marini $(\bowtie) \cdot$ R. Lapalombella $\cdot$ S. di Tullio $\cdot$ C. Marchionni Department of Histology, Embryology and Applied Biology, University of Bologna, Via Belmeloro, 8, Bologna, Italy

e-mail: marina.marini@unibo.it

M. Marini · A. Veicsteinas

Istituto Interuniversitario di Miologia, Chieti, Italy

E. Falcieri · S. Burattini

Institute of Morphological Sciences,

University of Urbino, Urbino, Italy

E. Falcieri

IGM-CNR, Istituti Ortopedici Rizzoli, Bologna, Italy

V. Margonato $\cdot$ F. Esposito $\cdot$ A. Veicsteinas

Institute of Physical Exercise, Health and Sport Activities,

University of Milan, Milan, Italy

D. Treré

Department of Experimental Pathology,

University of Bologna, Bologna, Italy

M. Samaja

Department of Medicine, Surgery and Dentistry,

University of Milan, Milan, Italy

A. Veicsteinas

Center of Sport Medicine, Don Gnocchi Foundation, Milan, Italy animals $(n=6)$ subjected to training followed by 4 -week detraining, the soleus/body weight ratio returned to normal, with only partial reversal of left ventricle capillarity and von Willebrand-positive cell density. Markers of angiogenesis (VEGF, KDR/VEGF-R2 and HIF- $1 \alpha$ mRNA, studied by real-time RT-PCR) were upregulated at the end of training, and returned to baseline value after detraining. Electron microscopy highlighted some morphological features in trained hearts (endothelial cell sprouting and bridges and pericyte detachment), suggestive of endothelial cell proliferation and capillary growth that were absent in untrained and detrained hearts. We conclude that the training-induced increase in cardiac capillarity and vascularization are retained for some time upon cessation of the training program even in the absence of angiogenic stimuli.

Keywords Angiogenesis - Exercise training · Cardioprotection $\cdot$ Rats $\cdot$ Electron microscopy

\author{
Abbreviations \\ VEGF Vascular endothelial growth factor \\ VEGF-R2 Vascular endothelial growth factor receptor 2 \\ bFGF Basic fibroblast growth factor \\ HIF-1 Hypoxia-inducible factor-1
}

\section{Introduction}

Regular practice of moderate aerobic exercise is widely recognized to be beneficial for the cardiovascular system (NIH 1995). Exercise training offsets many age-related gene expression changes (Bronikowski et al. 2003; Iemitsu et al. 2006), upregulates a number of cardioprotective and anti-oxidant proteins (Powers et al. 2004, Marini et al. 2007), improves contractile function and optimizes energy 
usage (Ventura-Clapier et al. 2007). Moreover, exercise increases the need for oxygen supply in the cardiac muscle, which may be met by the induction of neo-angiogenetic and vascular remodeling processes (White et al. 1998; Brown 2003); these, in turn, may trigger prevention of the adverse effects of coronary artery stenosis and occlusion (Schaper and Ito 1996).

The mechanisms underlying exercise-induced angiogenesis and vasculogenesis have been extensively reviewed in Conway et al. (2001), Gustafsson and Kraus (2001), Brown (2003), Djonov et al. (2003), Helisch and Schaper (2003), Kutryk and Stewart (2003), Prior et al. (2004), Bloor (2005) and Yoon et al. (2004). Despite general agreement about exercise-induced angiogenesis in skeletal muscle, myocardial angiogenesis is less established, especially after moderate exercise in post-pubertal animals (Hudlicka et al. 1992; Brown 2003). White et al. (1998) demonstrated in a swine heart model that capillary growth is limited only to the early phase of exercise training, and then gives rise to an increase in density and size of arterioles. By contrast, Efthimiadou et al. (2006) reported a significant increase in myocardial angiogenesis at the end of a 2-week intensive training protocol.

Nevertheless, little attention has been paid to the effects of interrupting exercise-training programs, an event which, in humans, may be produced by an array of causes including physical impairment and loss of motivation in pursuing a fitness program. For example, 4-8 weeks detraining in humans caused the endothelin-1 and nitric oxide to return to basal levels, these being two molecules involved in exercise-regulated signaling towards the vascular endothelium (Maeda et al. 2001). Furthermore, 2-4 weeks of detraining in rats regressed a number of cardiomyocyte parameters associated with aerobic fitness to pre-training values (Kemi et al. 2004) However, to the best of our knowledge, the effects of detraining on myocardial angiogenesis remain to be investigated.

To start investigating this issue, we used an in vivo model whereby rats were moderately trained (10 weeks, $\sim 60 \% V \mathrm{O}_{2 \max }$ ) (Marini et al. 2007) followed by 4 weeks of detraining to test the hypothesis whether the cessation of aerobic training could reverse training-induced myocardial angiogenesis. The 4-week detraining period was selected on the basis of the time required for an index of training, e.g., the soleus muscle/body weight ratio to return to the baseline value. To estimate the extension of the vascular network in the left ventricle, we evaluated three parameters: (1) visual examination of microscopic slides for capillarization and vascularization followed by semi-quantitative scoring, (2) manual counting of the capillaries in comparable sections photographed at the same enlargement, and (3) measurement of the size of the von Willebrand factor-positive area. Electron microscopy and mRNA expression data were also considered so as to evaluate ultrastructural changes and the involvement of angiogenetic mechanisms.

\section{Materials and methods}

Animals and training protocol

A total of 24 male albino Sprague-Dawley rats aged 9 weeks were placed in individual cages and fed a standard diet without limitations; room temperature was kept at $21 \pm 2^{\circ} \mathrm{C} ; 12 \mathrm{~h}$ of light was automatically alternated with $12 \mathrm{~h}$ of dark. After 1 week of acclimatization, 12 rats were randomly chosen to run on a six-lane rodent treadmill $1 \mathrm{~h} \mathrm{a}$ day, three times a week, at $10 \%$ grade slope. The speed was gradually increased to reach $25 \mathrm{~m} / \mathrm{min}$ in 5 weeks, which corresponds to $\sim 60 \% V^{2} \mathrm{O}_{2 \max }$ (Wisloff et al. 2001), then was maintained constant for a further 5 weeks. Control animals were placed on a non-moving treadmill during the training sessions. At the end of the 10-week training, six rats were randomly chosen for being killed immediately (trained group), while six were caged without exercise for a further 4 weeks before being killed (detrained group). Six sedentary rats (control group) were killed at the same time as the trained rats (at 19 weeks of age) and six at the same time as the detrained rats (at 23 weeks of age). All the experimental evaluations were carried out on all animals of each experimental group.

Animal handling, training protocol and mode of killing were approved by the Ethical Committee on the Use of Laboratory Animals of the Health Authority of Milan (Italy) according to the 86/609/CEE guidelines. Training was carried out according to the American Physiological Society guidelines for exercising rodents on treadmills (American Physiological Society 2006).

Rats were periodically examined by a veterinarian. Food consumption and body weight were evaluated three times a week. Their internal organs, examined by a pathologist on the day they were killed, appeared to be normal and disease-free.

Killing of the rats and heart conservation

The rats were anesthetized $(100 \mathrm{mg} / \mathrm{kg}$ ip heparinized sodium thiopental), weighed and then beheaded. The heart was removed; the blood was accurately drained before the heart was weighed on a precision scale. A $\sim 3 \mathrm{~mm}$ isodiametric fragment from the left ventricle apex was set apart for electron microscopy (see below), while the remaining part was cut into three pieces of the same size and immediately frozen in liquid nitrogen, then stored at $-80^{\circ} \mathrm{C}$ until use.

The soleus muscles were obtained from both the hind legs; they were blotted dry and freed of connective tissue 
before being weighed on a precision scale. Left and right soleus weight was averaged and related to the body weight.

\section{Light and electron microscopy}

The specimen was immediately minced into smaller $\left(<1 \mathrm{~mm}^{3}\right)$ fragments and quickly fixed with $2.5 \%$ glutaraldehyde in $0.1 \mathrm{M}$ phosphate buffer, postfixed with $1 \% \mathrm{OsO}_{4}$ in the same buffer, dehydrated with alcohol, and embedded in araldite. Semithin sections stained with $1 \%$ toluidine blue in distilled water at $60^{\circ} \mathrm{C}$ were selected on the basis of criteria supporting cross-sectioning (mainly myofibril spatial arrangement), taking care of sampling all fragments, in order to avoid biased selection. A total of 30 identical, nonoverlapping areas per sample, at $800 \times$ magnification, were digitalized and evaluated making use of the Olympus soft imaging system set up for the Philips CM10 electron microscope. The total surface evaluated per condition was $2.82 \mathrm{~mm}^{2}$. The cardiomyocyte cross-sectional area was evaluated by assessing the maximum and minimum diameter of all cardiomyocytes in the section area, which were averaged. The number of capillaries was evaluated following the visual identification of endothelial cells. Data were expressed as number of capillaries $/ \mathrm{mm}^{2}$. Thin sections, stained with uranyl acetate and lead citrate, were observed with the same electron microscope (Falcieri et al. 2000).

\section{Immunohistochemistry}

As stated above, the left ventricle was cut into three pieces of the same size before freezing. All pieces were sectioned with random orientation by a cryotome, and four $5 \mu \mathrm{m}$ thick sections/animal, obtained from different fragments, were chosen with the only criterion of avoiding sections with freezing defects. For the identification of endothelial cells, the sections were fixed in cold $4 \%$ paraformaldehyde, marked with rabbit anti-von Willebrand factor antibody (Dako, Denmark), followed by incubation with the antirabbit HRP-conjugated antibody and development of the chromogen reaction with 3,3'diaminobenzidine (DAB). The slides were assigned conventional numbers, so that the operator was blind to the training status of the rat. Under the light microscope, at $250 \times$ magnification, 30 non-overlapping fields were randomly selected for each section, avoiding areas with holes, folds, staining defects or large vessels. Images were acquired by a three CCD (charge-coupled device) color video camera (KY F55B, JVC Italia, Segrate MI, Italy) and examined with an image processing software (Image-Pro Plus, Media Cybernetics, Silver Spring, MD, USA). A specific function of the software allows the operator to interactively define the specific threshold, which permits automatic measurement of the structures intensely brown-stained, excluding any other structure less intensely stained in brown (background staining) or stained with other colors. By means of this system, for each microscopic field evaluated, the area stained with anti-von Willebrand factor antibody, identified by an intense brown staining and corresponding to the area covered by the endothelial cells present in the microscope field, was measured and expressed as a percentage of the total area. The total surface thus evaluated for each condition was $21.6 \mathrm{~mm}^{2}$.

In the same slides, the number of capillaries was evaluated by three independent investigators, who assigned a score (ranging from 1 to 3, low to high) to the overall capillarity and vascularization. The mean scores obtained by the three observers were then compared with the ANOVA test.

\section{Gene expression}

Total RNA was prepared after Trizol ${ }^{\mathrm{TM}}$ (Invitrogen) extraction from frozen left ventricle tissue and quality controlled, as previously described (Marini et al. 2007). Real-time RT-PCR was performed in an ABI PRISM 5700 real-time thermal cycler using the SYBR Green kit (Qiagen) and by normalizing to the housekeeping gene RPL13a. The primer sequences are reported in Table 1. . Primers were chosen with the help of the AMPLIFY free software; whenever possible, primers were designed so as to span an exon-exon junction and they were obtained from PROLIGO (Proligo, France SAS). Data were analyzed with the $2^{-\triangle \Delta C T}$ method (Livak and Schmittgen 2001), taking into account the efficiency of the real-time PCR reaction (Pfaffl 2001).

Table 1 Primer sequence and length of the amplicons of the genes studied with real-time RT-PCR

\begin{tabular}{lllll}
\hline UniGene & Gene name (symbol) & Left primer sequence & Right primer sequence & $\begin{array}{c}\text { Amplicon } \\
\text { length (nt) }\end{array}$ \\
\hline Rn 92211 & RPL13a & GATGAACACCAACCCGTCTC & CACCATCCGCTTTTTCTTGT & 175 \\
Rn 10852 & HIF-1alpha & AGCCTGGATCATGATGCTGT & ACTGTTTCCTTTTGAGCCGGTA & 192 \\
Rn 1923 & VEGF & ACCAAAGCCAGCACATAGGA & TTTCTCCGCTCTGAACAAGG & 192 \\
Rn 88869 & VEGF-R2/KDR/Flk1 & TGGTGTGTGGTCTTTTGGTG & GGTACATTTCTGGGGTGGTG & 154 \\
Rn 31808 & bFGF & CTCCAAGCAGAAGAGAGAGGA & CCAGGCGTTCAAAGAAGAAA & 135 \\
\hline
\end{tabular}


Statistical analysis

The differences among the groups were evaluated by the ANOVA test followed by the Tukey post-test. Values of $P<0.05$ were considered to be statistically significant.

\section{Results}

Effect of training and detraining on body, heart and muscle weights and evaluation of cardiac hypertrophy

At the end of training, the rats were leaner than the sedentary controls, with an increased soleus-to-body weight ratio, which shows that training was effective to increase the relative skeletal muscle mass (Table 2). Training did not affect either the absolute heart weight or the heart-tobody weight ratio. Furthermore, the cardiomyocyte crosssectional area, evaluated from semithin sections at $800 \times$ magnification, was the same in the control and the trained rats, thereby excluding onset of cardiac hypertrophy in this model. After detraining, both body and heart weights increased as expected (Hanson 2007), but the heart-to-body weight ratio and the cardiomyocyte cross-sectional area remained unchanged. By contrast, the soleus-to-body weight ratio returned to near the control value.

\section{Capillaries, endothelial cell count and vascularization}

To assess cardiac vascularization, we employed three methods, independent of each other, and applied to a fairly large number of experimental animals, sections and microscope fields. We were confident that this choice would allow an accurate assessment of angiogenesis and vascularization. First, three trained researchers examined immunostained (anti-von Willebrand factor antibody) slides, scoring the amount of stained areas on a 1-3 scale (minimum to maximum). Whereas sedentary controls received the lowest score, trained and detrained rats received a medium-to-high score (Fig. 1a).
Second, the number of capillaries per surface unit, or capillary density, was evaluated in toluidine-blue stained semithin sections of the left ventricle (Fig. 1b) with the aid of an Olympus imaging software. Representative images of toluidineblue stained semithin sections are shown in Fig. 2a-c. The 10-week mild exercise training doubled the capillary density, whereas detraining returned the density to near control value.

Third, the area occupied by endothelial cells was evaluated in anti-von Willebrand factor antibody-stained slides and expressed as a percentage of total area (Fig. 1c). Whereas training doubled this parameter with respect to sedentary controls, detraining did not have any appreciable effects. Representative images of immunohistochemically stained left ventricle sections are shown in Fig. 2d-f.

The reliability of our assesment of angiogenesis is supported by the high similarity of the results obtained using the three methods, although results reported in Fig. 1b were obtained with semithin sections from the left ventricle apex and those reported in Fig. 1a and c with sections from the remaining part of the ventricle.

\section{Electron microscopy}

Evidence of training-related cardiac neoangiogenesis, gathered from light microscopy and immunohistochemistry, was supported by electron microscopy observations. Thus, a very common finding in left ventricle samples from trained, but not from untrained or detrained subjects, is a sort of thin bridge, which appears to divide the capillary lumina and is presumably suggestive of intussusceptive growth or sprouting (Fig. 3). Figure 3b, shows a pericyte detached from the vessel wall, a finding which is generally associated with the vessel hyperdilatation that precedes the formation of new capillaries (Bergers and Song 2005).

\section{Gene expression}

Messenger RNA expression of VEGF, VEGF-R2 and HIF1 alpha, evaluated by real-time RT-PCR, was found significantly increased in the left ventricle samples of trained rats,

Table 2 Body and heart weight, cardiomyocyte cross-sectional area, heart-to-body and soleus-to-body weight ratio (mean \pm SD)

\begin{tabular}{lcccc}
\hline Sample & $\begin{array}{l}\text { Untrained control } \\
(19 \text { week-old })\end{array}$ & $\begin{array}{l}\text { Trained } \\
(19 \text { week-old })\end{array}$ & $\begin{array}{l}\text { Detrained } \\
(23 \text { week-old })\end{array}$ & $\begin{array}{l}\text { Detraining control } \\
(23 \text { week-old })\end{array}$ \\
\hline Body weight $(\mathrm{g})$ & $571.91 \pm 28.6$ & $541.44 \pm 29.7^{*}$ & $636.75 \pm 42.9^{\#}$ & $665.0 \pm 41.8$ \\
Heart weight $(\mathrm{g})$ & $1.39 \pm 0.08$ & $1.38 \pm 0.09$ & $1.55 \pm 0.19$ & $1.62 \pm 0.1$ \\
Heart weight/body weight $\times 1,000$ & $2.46 \pm 0.08$ & $2.56 \pm 0.12$ & $2.42 \pm 0.17$ & $2.44 \pm 0.15$ \\
Cardiomyocyte cross-sectional area $\left(\mu^{2}\right)$ & $27.24 \pm 2.07$ & $27.46 \pm 2.55$ & $27.21 \pm 1.62$ & Not evaluated \\
Mean soleus weight/body weigh $(\mathrm{mg} / \mathrm{g})$ & $0.36 \pm 0.03$ & $0.43 \pm 0.05^{*}$ & $0.38 \pm 0.03^{\#}$ & $0.34 \pm 0.04$ \\
\hline
\end{tabular}

* $P$ (trained vs. untrained control $) \leq 0.05$

\# $P$ (detrained vs detraining control) not significant 

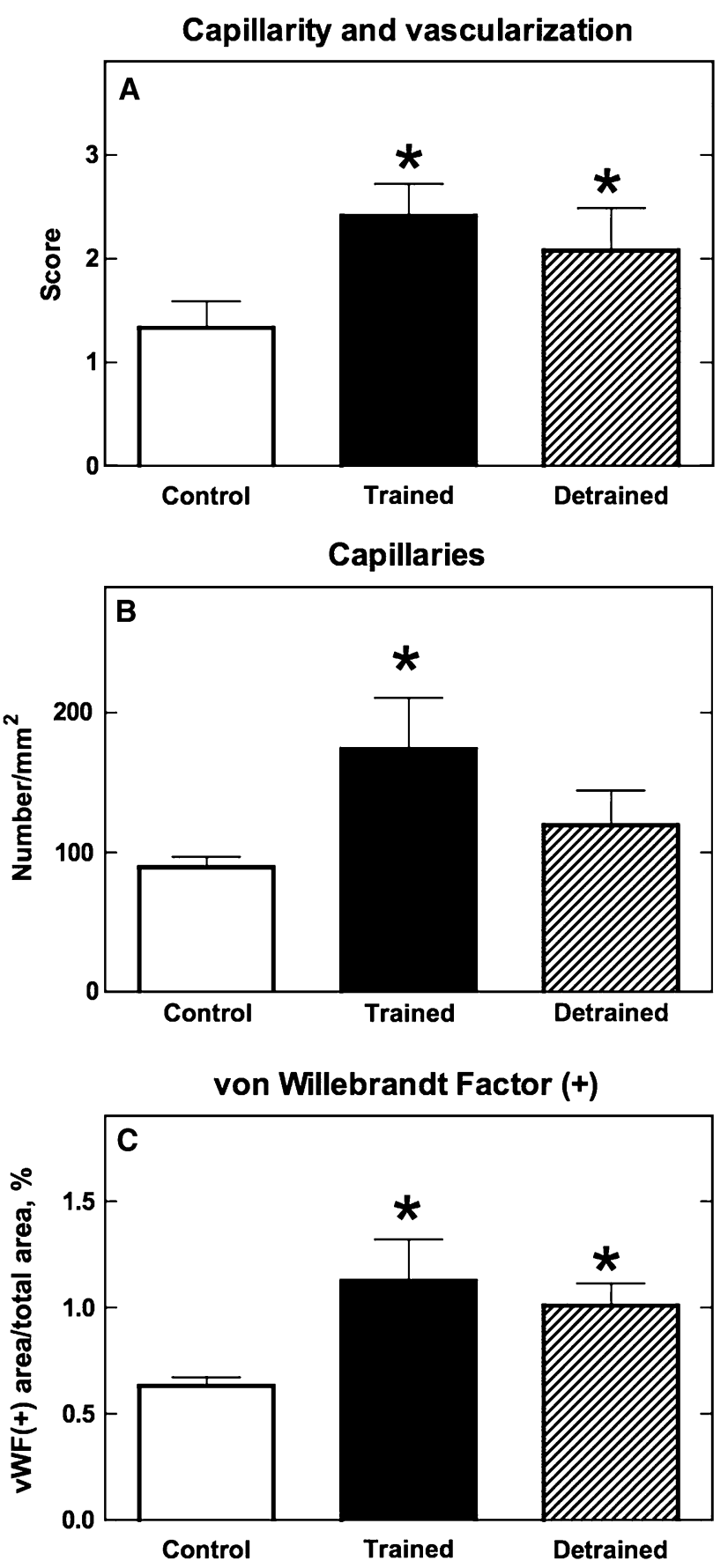

Fig. 1 Bar graph (mean \pm SD) showing left ventricle angiogenesis: a cryosections were immuno-stained with anti-von Willebrand factor antibody and examined by three investigators who scored the level of capillarity and vascularization on a scale of 1 (minimum) to 3 (maximum). b Capillaries were counted at $\times 800$ magnification in toluidine blue-stained semithin sections with the aid of an Olympus imaging software and their number was referred to a $1 \mathrm{~mm}^{2}$ area. c Immuno-stained (anti-von Willebrand factor antibody) images were evaluated at $\times 100$ magnification. The area corresponding to that covered by the endothelial cells was quantified by image analysis and expressed as a percentage of the total area. ANOVA yielded $P=0.0002,0.0002$ and $<0.0001$, respectively. $* P<0.05$ (Tukey post test) versus control whereas detraining reversed such training-induced increase in gene expression (Fig. 4). On the other hand, bFGF mRNA expression did not show any significant trainingrelated changes.

\section{Discussion}

In this study, we evaluated some parameters of myocardial angiogenesis in an in vivo model, whereby moderate exercise training did not induce cardiac hypertrophy, yet increased the relative skeletal muscle mass. Detraining restored the latter parameter. We showed that training increased myocardial angiogenesis concomitantly with upregulation of VEGF, KDR/VEGF-R2 and HIF-1 alpha. One month's detraining partially reversed the effects induced on angiogenesis by training, with complete reversal of the mRNA expression of angiogenetic factors.

Angiogenesis and vascular remodeling require the proliferation of endothelial and vascular smooth muscle cells, together with rearrangements and possibly expansion of the extracellular matrix. The neoformation of myocardial blood capillaries in swimming-exercised animals was initially reported by Ljungqvist and Unge (1973) and related to the resulting cardiac hypertrophy observed. Since then, conflicting reports have been published (Laughlin and Tomanek 1987; Hudlicka et al. 1992; White et al. 1998; Efthimiadou et al. 2006). As discussed by Brown (2003), such discrepancies may be due to the age of animals and to the timing of observations; for instance, it appears that exercise induces cardiac capillary growth, but this is rapidly followed by vascular remodeling, which leads to an increase in the number and size of arterioles (White et al. 1998). Thus, reports on exercise-induced angiogenesis, which focus on capillary density fail to convey the global picture of exercise-induced increase in cardiac blood supply.

In an attempt to overcome such limitations, we decided to employ the three approaches as described: (1) overall assessment of the status of tissue capillarity and vascularization; though subjective, this approach is very effective when applied by experienced workers blind to the identity of the slides; (2) evaluation of capillary density in semithin sections by morphometric analysis, the most widely used approach (see, for instance, Efthimiadou et al. 2006); since the training protocol adopted did not induce cardiac hypertrophy, the observed increase in myocardial capillary density corresponds to an increase in the capillary-to-fiber ratio as well; (3) evaluation of von Willebrand-positive endothelial cells in a semi-automated way by means of an image processing software. This approach allowed us to quantify the total vascular surface, including the arteriole area, thus 
Fig. 2 Toluidine-blue stained semithin sections $(\mathbf{a}-\mathbf{c})$ and immuno-stained (anti-von Willebrand factor antibody) cryosections showing endothelial cells $(\mathbf{d}-\mathbf{f})$. Sections from the left ventricles of untrained control $(\mathbf{a}, \mathbf{d})$, trained $(\mathbf{b}, \mathbf{e})$, detrained $(\mathbf{c}, \mathbf{f})$ rats. a-c $B a r=20 \mu \mathrm{m} ; \mathbf{d}-\mathbf{f}$ bar $=100 \mu \mathrm{m}$. Arrows in $\mathbf{a}-\mathbf{c}$ indicate endothelial cells
Fig. 3 Transmission electron microscopy images of left ventricle sections from trained rats. Arrows indicate cell bridges that partition pre-existing capillaries in the process of neoangiogenesis. In $\mathbf{b}, \mathrm{pc}=\mathrm{a}$ pericyte, caught in the act of detaching itself from a dilatating capillary, as described to occur in vessel remodeling.

a, $\mathbf{d}$ Bar $=2 \mu \mathrm{m} ; \mathbf{b}, \mathbf{f}$ bar $=0.5 \mu \mathrm{m} ; \mathbf{c}, \mathbf{e}$ bar $=1 \mu \mathrm{m}$
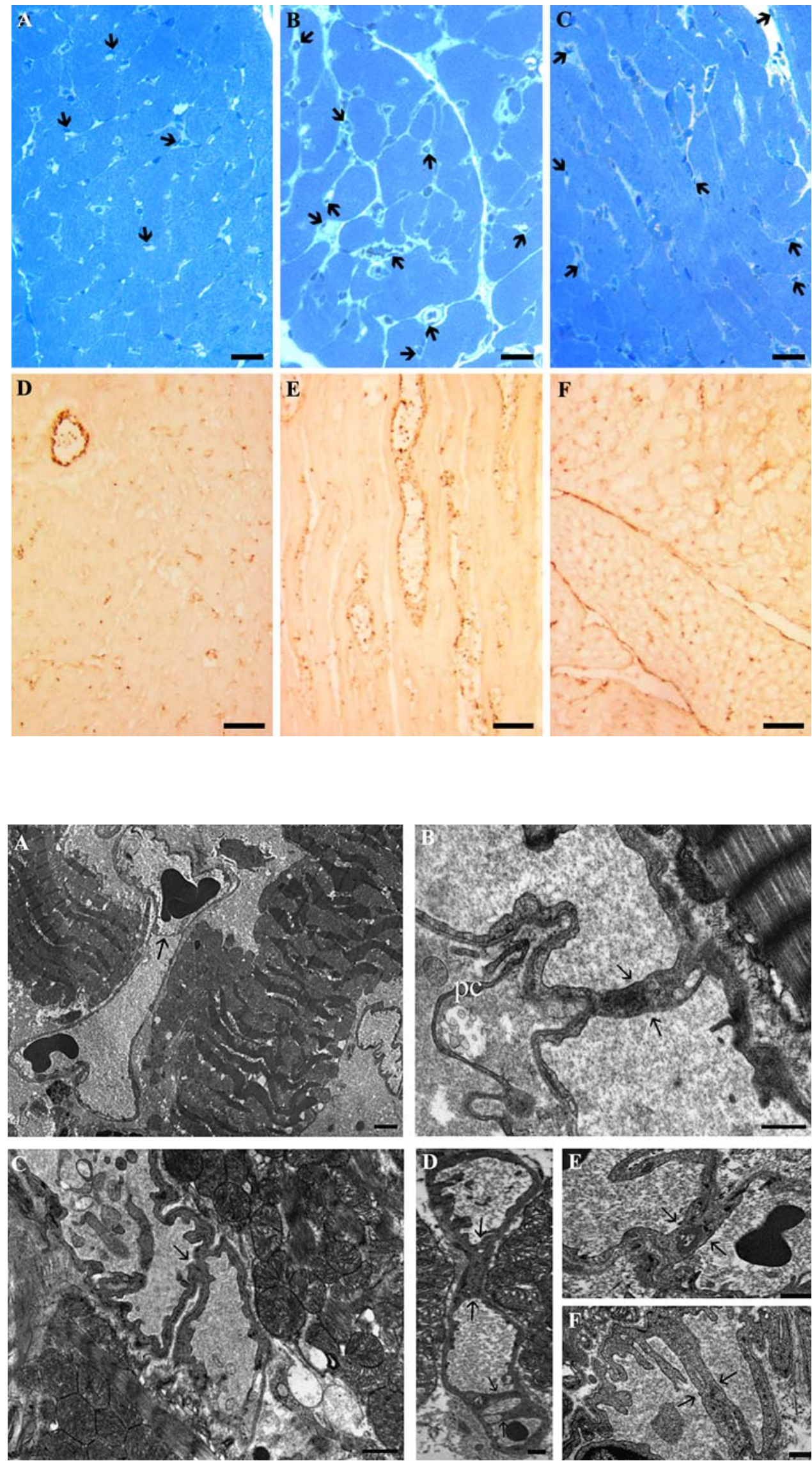
Fig. 4 Real-time RT-PCR: expression (mean $\pm \mathrm{SD}$ ) of some angiogenesis-related genes in rat left ventricles. Data were normalized to the housekeeping gene RPL13a. ANOVA yielded $P=<0.0001$ for all except bFGF (NS). * and \# indicate $P<0.05$ (Tukey post-test) versus control and trained, respectively
VEGF
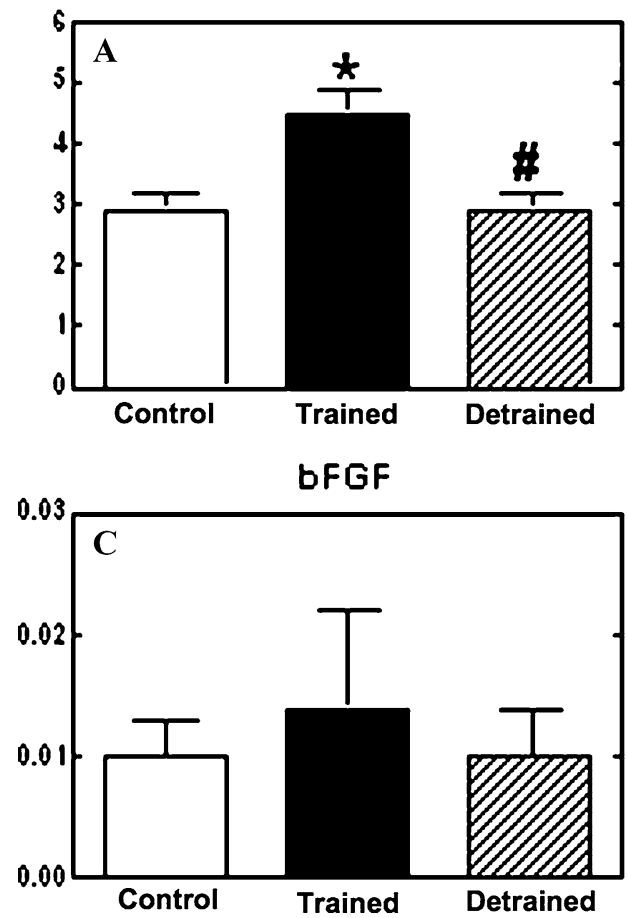

KDR
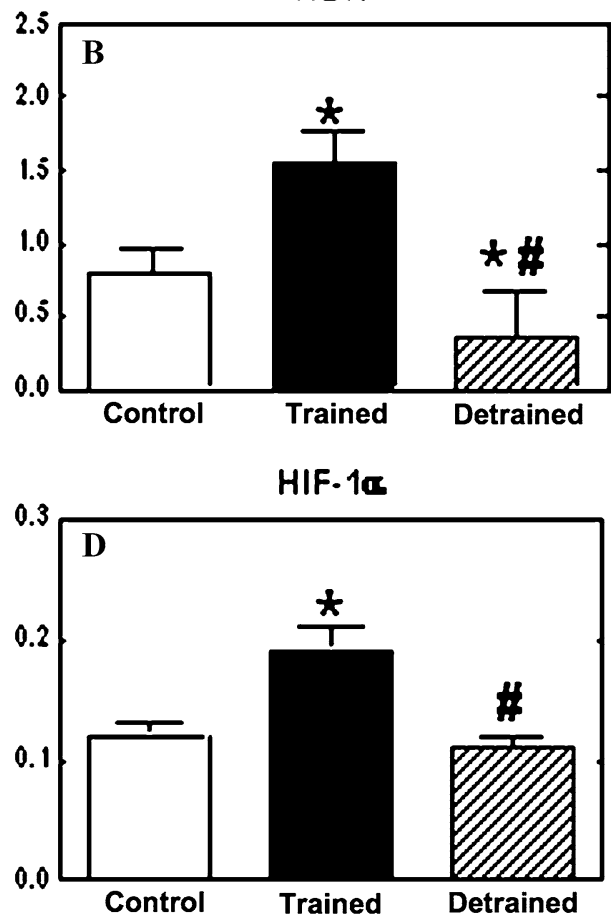

accounting not only for neo-angiogenesis (the de novo formation of capillary vessels), but also for transformation of capillaries into pre-capillary vessels and vascular remodeling by an increase in the diameter of pre-existing arterioles and venules. All these events have in common an increase in the number of endothelial cells present in the section to be examined. Although the sampling criteria did not strictly follow the methods suggested for stereological estimation of heart histological sections (Bruel and Nyengaard 2005), we judged that the chance of falling into the pitfalls of morphometry (Weibel et al. 2007) was minor because of random sampling and high number of counting events.

In summary, these approaches indicate that angiogenesis in the left ventricle does increase following a 10-week mild exercise training; however, this feature does not appear to be a permanent modification, becase it is subjected to a slow and partial reversal. Notably, electron microscopy lends support to light microscopy observations in showing training-induced angiogenesis.

It may be worth mentioning that the rats, which entered the study described here, were 9 weeks of age, i.e., young, sexually mature animals. Their weight at the end of the 10-week training program $(>550 \mathrm{~g}$, Table 2$)$ was higher than that of rats studied by Efthimiadou et al. (2006) (330$400 \mathrm{~g}$ ), which suggests that exercise-induced angiogenesis is not limited to young animals (Brown 2003). Moreover, no cardiac hypertrophy was induced in our study, as demonstrated by the absence of differences in the heart-to-body weight ratio and in the cardiomyocyte diameter between sedentary and exercised rats. This suggests that an increase in cardiac blood supply can be obtained with mild training protocols that do not induce extensive cardiac remodeling, a datum that may be relevant when different protocols of exercise-induced cardioprotection are evaluated for application in humans.

The effect of 4 weeks of detraining on the newly formed capillaries was undoubtedly that of a tendency to reversal, although the three approaches we adopted gave somewhat different results, with only the capillary count resulting in a statistically significant decrease. We believe that this may be due to an underestimation of the very small capillaries, bounded by a single endothelial cell, which are difficult to distinguish with aspecific staining, even if the observation is made at high magnification $(800 \times)$. This may suggest that reversal of neo-angiogenesis follows the inverse pathway of formation, with an initial reduction in their diameter before disappearing.

Exercise may generate repeated bursts of ischemia and hypoxia (Brown 2003) and, moreover, deeply affect the physical and hemodynamic forces (shear stress, pressure, stretch and compression). Altogether, these stimuli are likely to be the initiator events, directly or indirectly triggering the upregulation of a number of growth factors and of their cognate receptors on the target cells. Vascular endothelial growth factor (VEGF), and VEGF receptor 2 (KDR/Flk1) appear to be sufficient for the complete exercise-induced angiogenic response (Conway et al. 2001; Gigante et al. 2004; Bloor 2005). VEGF also upregulates the expression of matrix metalloproteinases, required for matrix remodeling and penetration of newly formed vessels 
(Wang and Keiser 1998). The role of VEGF in relation to the exercise-induced modifications of skeletal muscles, the mode of capillary growth (by intussusceptions rather than sprouting) and the mechanical forces involved have been thoroughly discussed by Brown and Hudlicka (2003). The real-time RT-PCR data reported here show that moderate exercise training upregulated VEGF and VEGF-R2, but not bFGF, expression. It has been shown that the intramuscular administration of bFGF increases myocardial angiogenesis (Efthimiadou et al. 2006); however, the present data suggest that exercise training exerts its angiogenic effect in the absence of bFGF upregulation. The finding that also HIF-1 alpha expression was increased is indicative of an involvement of hypoxia signaling in exercise-induced VEGF upregulation (Brown 2003). Thus, it is not surprising that the upregulation of VEGF signaling subsides upon cessation of stimulating messages. Long-term maintenance of the newly acquired vascularization would very likely require the persistence of positive stimulation.

Moderate exercise training shares some of the signaling pathways (Hearse 1994) and most of the induced array of antioxidant and protective molecules (Yellon and Downey 2003; Marini et al. 2007) with other preconditioning stimuli, such as repeated bursts of intense physical exercise. The results presented here show that, at variance with such stimuli, moderate exercise training also induces some anatomical adaptations to the increased need for oxygen supply, which may be relevant in providing cardioprotection even at a distant time from the training sessions. Future work will be devoted to the evaluation of training-induced cardioprotection in detrained animals.

The above-mentioned training-induced angiogenic and vascular remodeling are likely to ameliorate cardiac blood supply. It is worth noticing that they may be accomplished by adopting a moderate training protocol, which does not induce cardiac hypertrophy, and which are long-lasting. In fact, our data suggest that the exercise-induced modifications of myocardial microcirculation persisted, at least in part, for 4 weeks upon cessation of training, despite the fact that the expression of angiogenic factors returned to baseline. All animal exercise protocols derive their interest from the assumption that training may exert the same effects on rats and humans. However, we believe that it would be of little interest to study the time course of the detraining-induced reversal of angiogenesis in rats, given the differences in rat and people's life spans. It has been suggested that in adulthood, each rat month is roughly equivalent to 2.5 human years (Ruth 1935). If this is true, the partial persistence of training-induced angiogenesis in the rat after 4 weeks of detraining may contribute to motivating people's practice of moderate aerobic training.
Acknowledgments This study was supported by grants from the Italian Ministry of University and Research (PRIN 2004054720) and from the Cariplo Foundation (2005 Project).

\section{References}

American Physiological Society (2006) Resource book for the design of animal exercise protocols, February 2006. http://www.theaps.org/pa/action/exercise/book.pdf

Bergers G, Song S (2005) The role of pericytes in blood-vessel formation and maintenance. Neuro Oncol 7:452-464

Bloor CM (2005) Angiogenesis during exercise and training. Angiogenesis 8:263-271

Bronikowski AM, Carter PA, Morgan TJ, Garland T, Ung N, Pugh TD, Weindruch R, Prolla TA (2003) Lifelong voluntary exercise in the mouse prevents age-related alterations in gene expression in the heart. Physiol Genomics 12:128-138

Brown MD (2003) Exercise and coronary vascular remodelling in the healthy heart. Exp Physiol 88:645-658

Brown MD, Hudlicka O (2003) Modulation of physiological angiogenesis in skeletal muscle by mechanical forces: involvement of VEGF and metalloproteinases. Angiogenesis 6:1-14

Bruel A, Nyengaard JR (2005) Design-based stereological estimation of the total number of cardiac myocytes in histological sections. Basic Res Cardiol 100:311-319

Conway EM, Collen D, Carmeliet P (2001) Molecular mechanisms of blood vessel growth. Cardiovasc Res 49:507-521

Djonov V, Baum O, Burri PH (2003) Vascular remodeling by intussceptive angiogenesis. Cell Tissue Res 314:107-117

Efthimiadou A, Asimakopoulos B, Nikolettos N, Giatromanolaki A, Sivridis E, Lialiaris TS, Papachristou DN, Kontoleon E (2006) The angiogenetic effect of intramuscular administration of b-FGF and a-FGF on cardiac muscle: The influence of exercise on muscle angiogenesis. J Sports Sci 24:849-854

Falcieri E, Luchetti F, Burattini S, Canonico B, Santi S, Papa S (2000) Lineage-related sensitivity to apoptosis in human tumor cells undergoing hyperthermia. Histochem Cell Biol 113:135-144

Gigante B, Tarsitano M, Cimini V, De Falco S, Persico MG (2004) Placenta growth factor is not required for exercise-induced angiogenesis. Angiogenesis 7:277-284

Gustafsson T, Kraus WE (2001) Exercise-induced angiogenesis-related growth and transcription factors in skeletal muscle, and their modification in muscle pathology. Front Biosci 6:75-89

Hanson A (2007) How old is a rat in human years. Rat Behav Biol. http://www.ratbehavior.org/rats.html

Hearse DJ (1994) Myocardial ischaemia: can we agree on a definition for the 21st century? Cardiovasc Res 28:1737-144

Helisch A, Schaper S (2003) Arteriogenesis: the development and growth of collateral arteries. Microcirculation 10:83-97

Hudlicka O, Brown MD, Egginton S (1992) Angiogenesis in skeletal and cardiac muscle. Physiol Rev 72:369-417

Iemitsu M, Maeda S, Jesmin S, Otsuki T, Miyauchi T (2006) Exercise training improves aging-induced downregulation of VEGF angiogenic signaling cascade in hearts. Am J Physiol Heart Circ Physiol 291:H1290-H1298

Kemi OJ, Haram PM, Wisloff U, Ellingsen O (2004) Aerobic fitness is associated with cardiomyocyte contractile capacity and endothelial function in exercise training and detraining. Circulation 109:2897-904

Kutryk MJB, Stewart DJ (2003) Angiogenesis of the heart. Microsc Res Tech 60:138-158

Laughlin MH, Tomanek RJ (1987) Myocardial capillarity and maximal capillarity diffusion capacity in exercise-trained dogs. J Appl Physiol 63:1481-1486 
Livak JK, Schmittgen TD (2001) Analysis of relative gene expression data using real-time quantitative PCR and the $2^{-\triangle \Delta C T}$ method. Methods 25:402-408

Ljungqvist A, Unge G (1973) The proliferative activity of the myocardial tissue in various form of experimental cardiac hypertrophy. Acta Pathol Microbiol Scand Sect A 81:233-240

Maeda S, Miyauchi T, Kakiyama T, Sugawara J, Iemitsu M, Irukayama-Tomobe Y, Murakami H, Kumagai Y, Kuno S, Matsuda M (2001) Effects of exercise training of 8 weeks and detraining on plasma levels of endothelium-derived factors, endothelin-1 and nitric oxide, in healthy young humans. Life Sci 69:1005-1016

Marini M, Lapalombella R, Margonato V, Ronchi R, Samaja M, Scapin C, Gorza L, Maraldi T, Carinci P, Ventura C, Veicsteinas A (2007) Mild exercise training, cardioprotection and stress gene profile. Eur J Appl Physiol 99:503-510

National Institutes of Health Physical activity and cardiovascular health (1995) NIH Consensus Development Conference Statement 13:11-22

Pfaffl MW (2001) A new mathematical model for relative quantification in real-time RT-PCR. Nucl Acids Res 29:e45

Powers SK, Quindry J, Hamilton K (2004) Aging, exercise, and cardioprotection. Ann N Y Acad Sci 1019:462-470

Prior BM, Yang HT, Terjung RL (2004) What makes vessels grow with exercise training? J Appl Physiol 97:1119-1128
Ruth EB (1935) Metamorphosis of the pubic symphysis. I. The white rat (Mus norvegicus albinus). Anat Rec 64:1-7

Schaper W, Ito W (1996) Molecular mechanisms of coronary collateral vessel growth. Circ Res 79:911-919

Ventura-Clapier R, Mettauer B, Bigard X (2007) Beneficial effects of endurance training on cardiac and skeletal muscle energy metabolism in heart failure. Cardiovasc Res 73:10-18

Wang H, Keiser JA (1998) Vascular endothelial growth factor upregulates the expression of matrix metalloproteinases in vascular smooth muscle: role of flt- 1 . Circ Res 83:832-840

Weibel ER, Hsia CCW, Ochs M (2007) How much is there really? Why stereology is essential in lung morphometry. J Appl Physiol 102:459-467

White FC, Bloor CM, McKirnan MD, Carroll SM (1998) Exercise training in swine promotes growth of arteriolar bed and capillary angiogenesis in heart. J Appl Physiol 85:1160-1168

Wisloff U, Helgerud J, Kemi OJ, Ellingsen O (2001) Intensity-controlled treadmill running in rats: $\mathrm{VO}(2 \mathrm{max})$ and cardiac hypertrophy. Am J Physiol Heart Circ Physiol 280:H1301-H1310

Yellon DM, Downey JM (2003) Preconditioning the myocardium: from cellular physiology to clinical cardiology. Physiol Rev 83:1113-1151

Yoon YS, Johnson IA, Park JS, Diaz L, Losordo DW (2004) Therapeutic myocardial angiogenesis with vascular endothelial growth factors. Mol Cell Biochem 264:63-74 